\title{
On the Management of Virtual Organizations' Dissolution (in Virtual Business Networks)
}

\author{
Nicolás Hormazábal ${ }^{1}$ and Josep Lluís de la Rosa ${ }^{2}$ \\ ${ }^{1}$ Tecnalia, Paseo Mikeletegi, 7 - Parque Tecnológico E 20009, San Sebastián, Spain \\ nicolash@eia.udg.edu \\ ${ }^{2}$ Agents Research Lab, Edifici PIV, Campus de Montilivi, 17071. Girona, Spain \\ peplluis@eia.udg.edu
}

\begin{abstract}
The dissolution of Virtual Organizations is not just a stage where the commitments between the partners take to an end, but an issue that is worth considering through all the life cycle in Virtual Organizations. This paper gives further light to the model of Virtual Organizations split in several phases, detailing their roles and significance, and explaining from previous experiences why the dissolution has to be carefully planned fairly in advance. The key elements for managing the dissolution of virtual organizations are described, and further evidence on how they can have positive influence to the performance of Virtual Organizations, are contributed highlighting the phase's significance.
\end{abstract}

Keywords: Virtual Organization, Dissolution, Business Communities, Business Networks.

\section{Introduction}

Business Communities (BC) as non-hierarchical collaborative environments allow companies to create new Virtual Organizations (VO) as new business opportunities arise, providing tools for knowledge and resources sharing, a trusted network of companies and means for collaboration among the $\mathrm{BC}$ members in order to respond the market needs for competing with larger companies. Collaboration and knowledge sharing is of high added value that is key in these collaborative environments as companies can benefit from the knowledge and lessons learned from past experiences when creating new VOs.

In the state of the art, typically three main different phases have been defined in the VOs' lifecycle: Form, Operate and Dissolve [1], with a fourth additional one, Evolve [2] where the VO can modify its infrastructure and cooperation agreements in order to better respond to environmental changes or performance issues. The formation and operation phases have been extensively studied among the current literature, but the dissolution phase has been addressed in a superficial way. Dissolution is a phase that is performed only during the last steps of the VO lifecycle, but its significance ranges the whole lifecycle in different ways yet to be understood that deserve further study. 
Part of this work is included in the methodology design created for the NetChallenge European project ${ }^{1}$ [Ref.: FP7-CP-FP229278-2]. The paper is organized as follows: Section 2 focuses on the dissolution phase's significance by itself, section 3 emphasizes the significance of the dissolution among the other phases and section 4 concludes and explains the future work.

\section{Dissolution Phase}

During the dissolution phase, some issues must be addressed such as the management of results from the collaboration process made through the VO lifecycle like the intellectual property rights over the production, liabilities towards the customer (warranties), and financial results. Most of this should have been already defined and regulated in the cooperation agreement made during the formation phase, but within a $\mathrm{BC}$ environment, there should be also some other issues to be considered for the dissolution.

The dissolution phase represents a unique opportunity to retrieve, store and share the lessons learned from the cooperation and interaction between the different companies that participated in the VO, providing valuable information for future $\mathrm{VO}$ creation and operation.

\subsection{Information Sharing during Dissolution}

When creating a new collaboration network for VOs such as a Business Community (BC), one of the basic concepts that must be considered to assure collaboration and knowledge sharing is a common information and data structure properly modeled and organized [4] for facilitating the access to the information. One of the most valuable pieces of information is the one retrieved at the moment of reviewing past performed activities (it is usually named "post-mortem" in software projects), as a way to learn lessons from the experience. The lessons learned the hard way (by empirical failures), can be opportunities in future projects [5]. Creating a context that makes organizational learning possible from the past experiences represents a powerful tool for better future decision making.

The information stored during the VO dissolution as a way for future reference can be divided into two groups: Performance Review and Dissolution Cause Review.

Performance Review: Some of the information that should be modeled when creating a Business Community is the Key Performance Indicators (KPI), which must be defined before any VO should be created to allow comparing performance indicators from different organizations. The assessment of the KPIs is certifying the status of a VO, whether it is on the right track or not. The possible KPIs for a VO can be varied from the one VO to another VO but some are commonly used as lead-time, time-to-market, resource utilization, annual turnover, customer satisfaction level, business growth, etc. The KPI can have different values in respect to business strategy such as KPI for BC, VO, marketing, manufacturing, supply chain management, etc.

${ }^{1}$ http: / / www. netchallenge.org/ 


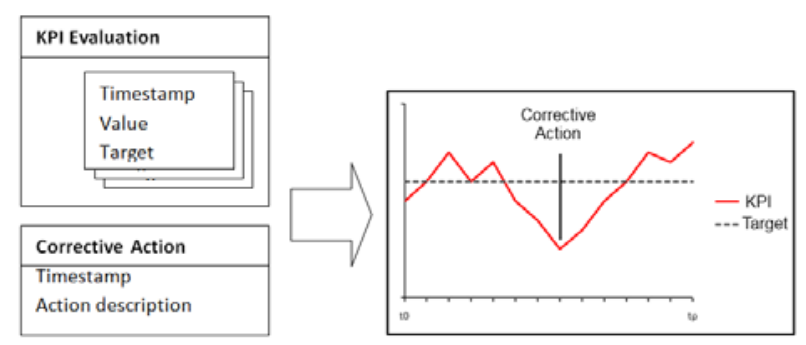

Fig. 1. KPI Evaluations: KPIs are evaluated and compared against the target value

Most of the KPI should have been evaluated during the VO operation as part of a VO performance monitoring process, but there are others that can only be evaluated after the collaboration has finished. These indicators are related to the individual assessment of the VO made by each partner, and may be subjective depending on their expectations, such as the partners' commitment evaluation, collaboration level or communication. This information should help to better define the success or failure of the overall VO, as it goes beyond of the fact whether the VO has achieved its main objectives or not. A VO that has achieved its goals but has gone through major internal problems and has needed many corrective actions should not be considered as equally successful as a VO that has achieved its goals without significant issues.

The KPI's historical data should be compiled along the stream of corrective actions executed, if any. This will help for future reference about the efficiency on the actions taken on the VO based on its impact to the performance indicators.

Each action should be documented as it will be useful for the future VOs in case they find similar cases; it is an important asset regarding the lessons learned point of view. So the evaluation for each KPI should contain a timestamp of the evaluation, the value and the desired target defined by the initial VO commitments and goals. The corrective actions (if any) should also include a timestamp to help establishing a connection among the actions and the KPIs performance changes. From Figure 1, it is observed that the performance level for each KPI can be measured by its deviation from its target value: If a VO has failed to achieve target product delivery deadline (KPI) corrective actions can be to revise the production strategy collaboratively in terms of resource reallocation, possible penalty for delayed partner(s), increasing the level of trust and networking among VO partners, improve partners' capability, etc.

Dissolution Cause Review: Usually, the dissolution is triggered when the VO has fulfilled its goals, or the business opportunity the VO was created for, no longer exists. But there are other causes that could make a VO dissolve, such as performance problems, environmental changes, internal VO members' decisions or resources shortage [3].

As additional information to the performance review, the dissolution cause should be included. It is expected that it puts in context the dissolution cause (why this cause was triggered, in case there were related KPIs), helping to detect in the future what consequences could be derived from performance deviations. 


\subsection{Product Support After Dissolution}

VOs usually are created having a product or service development as an objective (after a business opportunity has been detected). The support tasks like after-sales actions, warranties and liabilities over components or process are not always part of the main VO goals. In the cooperation agreement the liabilities over the VO results should be defined, and these have to be assigned once the VO starts its dissolution process for assuring support towards the customers once the VO has been dissolved.

Some approaches suggest that the liabilities of each component of a product should be related to the component's suppliers [2], but the problem here is that the connection between the customer and the support organization goes from a one to one relation (the customer and the $\mathrm{VO}$ ) to a one to many (the customer and each one of the suppliers). This could be a problem in terms of information management and product/technical support tracking as the support actions and liabilities are scattered among the different suppliers.

Another approach would be to provide support during the VO lifespan (mainly in the operation phase), and thus the VO should be kept active until no support is any longer needed. But this means to keep active a VO even if the business opportunity no longer exists and no production or activity of any kind is being performed besides the occasional support tasks, having members and resources assigned that wouldn't be needed, as not every partner should have been involved in production operations that would need further support.

Finally, as a way to keep the relation between the customer and the support team as a one to one relationship, allowing to each former VO member to be in knowledge of the support actions, the partners can create a new organization only focused on providing after-sales and technical support. Some approaches suggest having a specific virtual organization for after-sale services which should also provide quick services activities [6].

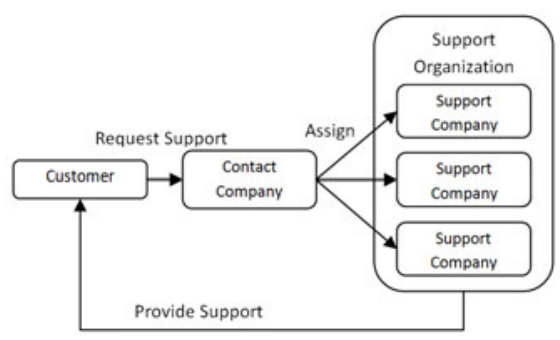

Fig. 2. Example of a support organization

Using this last approach as reference, the VO can define a "contact" company which will be the one who will be facing the customer inquiries and support requests, and will then delegate them to the respective partners (Fig 2); we will name this structure "support organization". This way, the relationship towards the customer remains almost equal than when the VO was active. This support organization should have less resources assigned, allowing the $\mathrm{BC}$ to count on more free resources to face new business opportunities and the components liabilities are still remaining with 
their suppliers (only there is a proxy between the customer and the companies now). The support organization's structure and activities should be simpler than a VO, as no production is needed but only coordination between its members for the support actions, and the resources committed from every company towards the support tasks should be significantly lower than the committed towards the dissolved VO.

This support organization should be kept active until no longer support is needed (for example, when warranties periods have expired).

\subsection{Additional Dissolution Tasks}

Additional tasks, more related to the initial commitments made during the VO formation should be performed during the dissolution. These tasks include sharing out the assets and financial results, intellectual property rights assignment and formalization, and the identification and performing of additional pending tasks that could be left from the operate phase (in case the VO has been dissolved unexpectedly and commitments towards external entities, such as suppliers, are left open).

During VO dissolution, the information access rights must be defined, specifying which information each partner can access to, and the security measures that will be used for protecting this information (encryption, passwords, etc.). The access rights level should specify if partners could use this information for forming and operating future $\mathrm{VO}$ in case a third party is involved.

\section{Dissolution and the Other VO Life-Cycle Phases}

The dissolution phase has an important role on the other VO life-cycle's phases, mainly from the knowledge acquired during the dissolution phase, but also as an issue to be considered when performing the task related to each of these phases.

The dissolution phase should provide information that could be used during the other life-cycle phases, but also is a key phase that needs to be considered from the beginning of a VO for avoiding further difficulties when reaching the final lifecycle's phase. The VO's cooperation agreement must include some key elements that are needed for the dissolution, which mainly are:

- Conditions and guidelines for the final financial statement: Detailing the initial assets, agreed liabilities, share out conditions, etc.

- Intellectual property rights: When creating the VO, the IPR of each partner must be decided over the results from the organization.

- Dissolution conditions: If a VO is created with a fixed lifespan (e.g. if it provides a service for a limited season), it must be defined at the initial cooperation agreement. The needed votes for VO dissolution (in case there is no dissolution agreement) and other dissolution conditions must be detailed.

- Privacy over the shared information: The members must decide at which level of detail the information will be shared with the BC (e.g. in the case of the performance evaluations some VOs would prefer to detail only the percentage over the target KPI value, instead of absolute values). 
- Dissolution process agreement: Any other needed step, which depends on each VO's scope, for the dissolution must be detailed, creating the partners' agreement for dissolution.

Most of these elements depend on the $\mathrm{BC}$, which could restrict them or provide a template for the cooperation agreement including standard dissolution elements. These elements could change if the VO is modified with the agreement of the VO partners. On the other side, the dissolution's results (performance reports, dissolution cause identification), support in different ways the other VO life-cycle phases.

\subsection{Form Phase}

When creating a new VO, at the form phase companies select partners that fit better the needs of the business opportunity using mainly the information each company gives about itself, its capabilities such as resources, availability or knowledge and expertise. One of the main benefits of a $\mathrm{BC}$ as an environment for creating new VOs, is that the companies that belong to it usually have already been profiled and their competencies identified at the moment of joining the community [7] (i.e. within a qualification process). A qualification process verifies the capabilities of the companies before joining a $\mathrm{BC}$, but the information obtained from this process does not necessarily reflect the cooperation capacities of a company in a real performing VO. In cases that the $\mathrm{BC}$ has been active for a large period of time, it is likely that companies already had previous interactions with other BC members (and thus they have empirical knowledge about their performance in different situations).

The performance reports made during the dissolution phase provide information from real interaction within a VO. This information represents not only quantitative information about the member's performance, but also qualitative information that should be able to generate a list of trusted candidate partners in different ways, ranked by trust, reliability and other information from previous interactions. In order to take advantage of this information, $\mathrm{BC}$ members should have a set of visibility rights to the information related the other members' capacities when creating a new VO [4].

\subsection{Operate Phase}

One critical task related to the dissolution during the operate phase, is the identification of a dissolution cause. Dissolution not always happens when a business opportunity does not exist anymore, but other causes must be considered, such as unexpected events based on environmental changes, or internal problems in the VO: A key $\mathrm{VO}$ member could leave the $\mathrm{VO}$ and thus reducing the resources to a point that maybe the objectives could not be achieved, or unexpected environmental changes that affect the business opportunity could happen among other situations. Any unexpected cause that could lead the VO to its dissolution should be considered for future reference in order to better respond to future events or observed performance decreases [3]. If a dissolution cause is not properly detected, it could end in an underperforming $\mathrm{VO}$ that maybe wouldn't be available to achieve its goals. In this case, the VO should decide if it needs a reconfiguration (or evolution) to better respond, or in the worst case, dissolve itself. 
BCs have an overall capacity that should be enough to respond to the market demands and position itself in the desired market position, based on the total capabilities of each of its members. When new business opportunities arise, new VOs are created and their members' resources are committed to it, reducing the BC available resources. Properly timed dissolution causes identification could free valuable resources for the whole $\mathrm{BC}$ that in other cases could be committed to an underperforming $\mathrm{VO}$ [8].

The knowledge acquired during the dissolution phase should also support the evolution of the VO. Once performance issues are detected in the different measured KPIs, the knowledge base should be able to provide information from past performance reports and the actions taken back then with their results. The identification of similar past cases is out of the scope of this paper, and please refer to tools using methodologies like case based reasoning [3] which is a good example of an automated tool for recommending actions for performance issues based on past experiences, or even recommending the dissolution if the results show that probably none of the VO objectives can be achieved given its current status. The dissolution phase should be able to provide enough information to support these kinds of tools.

\section{Conclusions and Future Work}

Dissolution represents a phase in the VO life-cycle and other collaboration forms that usually is overlooked, being for a long time mentioned as the least studied phase of the life-cycle [9], [10].

In this paper we tried to give arguments to show the dissolution phase as an opportunity to create better VOs in the future, which should be prepared to better respond to unexpected issues and situations in the future by providing past experiences knowledge. Plus, at a higher level, a dissolution cause identified at the right time, could help the whole $\mathrm{BC}$ by freeing inactive resources assigned in low performing VOs. The dissolution phase then, besides finishing the formal actions for closing the commitments between the VO partners, must collect and store information about the VO performance in a reusable, structured way in order to have it available for future reference.

In this paper we have identified the contributions of the dissolution phase such as:

- Support for VO partners search and selection.

- Support for VO performance improvement.

- Reference for past VO actions for performance improvement.

Plus, we identified where the dissolution must be considered and planned:

- During the formation phase for the definition of the dissolution conditions.

- VO evolution, in case the VO has changed its initial commitments, or added new partners.

And what should be considered during the dissolution phase:

- VO dissolution causes identification for future reference.

- Support organizations creation after VO dissolution.

- Creation of Performance Reports. 
Future work is focused mainly on the complete formalization of the dissolution process and its steps (as well the formalization of a support organization creation and structure), and detailing further the significance of this phase by providing more proofs through experimentation and simulation environments.

Acknowledgments. The authors would like to acknowledge the co-funding of the European Commission within NMP priority of the Seventh RTD Framework Programme (2007-13) for the Net Challenge project (Innovative Networks of SMEs for Complex Products Manufacturing), Ref. CP-FP 229287-2.

\section{References}

1. Strader, T.J., Lin, F.-R., Shaw, M.J.: Information infrastructure for electronic virtual organization management. Decision Support Systems 23(1), 75-94 (1998)

2. Camarinha-Matos, L.: Infrastructures for virtual organizations - where we are, vol. 2, pp. 405-414 (2003) (presented at the ETFA 2003 - 9th Int. Conf. on Emerging Technologies and Factory Automation, Lisboa, 2003)

3. Hormazábal, N., Cardoso, H.L., De La Rosa, J.L., Oliveira, E.: An approach for virtual organisations' dissolution. In: Padget, J., Artikis, A., Vasconcelos, W., Stathis, K., da Silva, V.T., Matson, E., Polleres, A. (eds.) COIN@AAMAS 2009. LNCS, vol. 6069, pp. 70-85. Springer, Heidelberg (2010)

4. Afsarmanesh, H., Camarinha-Matos, L.M.: A Framework for Management of Virtual Organization Breeding Environments. In: Proceedings of IMP Group Conference, pp. 35$48(2005)$

5. Collier, B., DeMarco, T., Fearey, P.: A defined process for project post mortem review. IEEE Software 13(4), 65-72 (1996)

6. Hartel, I.: Virtual Organization of After-Sales Service in the One-Of-A-Kind Industry. In: Proceedings of the IFIP TC5/WG5.5 Third Working Conference on Infrastructures for Virtual Enterprises: Collaborative Business Ecosystems and Virtual Enterprises, PRO-VE 2002, p. 656 (2002)

7. Camarinha-Matos, L.M., Afsarmanesh, H., Ollus, M.: ECOLEAD: A holistic approach to creation and management of dynamic virtual organizations. In: PRO-VE 2005 Collaborative Networks and their Breeding Environments, vol. 186, pp. 3-16. Springer, Boston (2005)

8. Hormazábal, N., De la Rosa, J.L.: Virtual Organisations Dissolution. In: Proceedings of the Distributed Computing and Artificial Intelligence, Advances in Soft Computing, vol. 79, pp. 139-146 (2010), doi:10.1007/978-3-642-14883-5_18

9. Muntaner-Perich, E., De La Rosa Esteva, J.L.: Towards a formalisation of dynamic electronic institutions. In: Sichman, J.S., Padget, J., Ossowski, S., Noriega, P. (eds.) COIN 2007. LNCS (LNAI), vol. 4870, pp. 97-109. Springer, Heidelberg (2008)

10. Camarinha-Matos, L.M., Afsarmanesh, H.: Tendencies and General Requirements for Virtual Enterprises. In: Proceedings of the IFIP TC5 WG5.3 / PRODNET Working Conference on Infrastructures for Virtual Enterprises: Networking Industrial Enterprises, pp. 15-30 (1999) 